\title{
Total prompt $\gamma$-ray emission in fission
}

\author{
C.Y. Wu ${ }^{1, a}$, A. Chyzh ${ }^{1,2}$, E. Kwan ${ }^{1,3}$, R.A. Henserson ${ }^{1}$, T.A. Bredeweg ${ }^{4}$, R.C. Haight ${ }^{4}$, A.C. Hayes- \\ Sterbenz ${ }^{4}$, H.Y. Lee ${ }^{4}$, J.M. O'Donnell ${ }^{4}$, and J.L. Ullmann ${ }^{4}$ \\ ${ }^{1}$ Lawrence Livermore National Laboratory, Livermore, California 94550 USA \\ ${ }^{2}$ North Carolina State University, Raleigh, North Carolina 27695 USA \\ ${ }^{3}$ National Superconducting Cyclotron Laboratory, East Lansing, Michigan, 48824 USA \\ ${ }^{4}$ Los Alamos National Laboratory, Los Alamos, New Mexico 87545 USA
}

\begin{abstract}
The total prompt $\gamma$-ray energy distributions for the neutron-induced fission of ${ }^{235} \mathrm{U}$, ${ }^{239,241} \mathrm{Pu}$ at incident neutron energy of $0.025 \mathrm{eV}-100 \mathrm{keV}$, and the spontaneous fission of ${ }^{252} \mathrm{Cf}$ were measured using the Detector for Advanced Neutron Capture Experiments (DANCE) array in coincidence with the detection of fission fragments by a parallel-plate avalanche counter. DANCE is a highly segmented, highly efficient $4 \pi \gamma$-ray calorimeter. Corrections were made to the measured distribution by unfolding the two-dimension spectrum of total $\gamma$-ray energy vs multiplicity using a simulated DANCE response matrix. The mean values of the total prompt $\gamma$ ray energy, determined from the unfolded distributions, are $\sim 20 \%$ higher than those derived from measurements using single $\gamma$-ray detector for all the fissile nuclei studied. This raises serious concern on the validity of the mean total prompt $\gamma$-ray energy obtained from the product of mean values for both prompt $\gamma$-ray energy and multiplicity.
\end{abstract}

\section{Introduction}

The total prompt $\gamma$-ray emission in fission accounts for about $40 \%$ of the total energy released by $\gamma$ ray emission that makes up about $10 \%$ of the total energy released in reactor core. The heating in nuclear reactors attributed to the total $\gamma$-ray emission in fission is underestimated up to $28 \%$ using the evaluated data for the main reaction channels, ${ }^{235} \mathrm{U}(\mathrm{n}, \mathrm{f})$ and ${ }^{239} \mathrm{Pu}(\mathrm{n}, \mathrm{f})$ [1]. This discrepancy is significantly greater than $7.5 \%$, an upper bound of the uncertainty deemed necessary to adequately model the heat deposit in the fuel core $[2,3]$. Therefore, efforts are needed to improve the experimental data on the $\gamma$-ray emission in fission. As a matter of fact, the request for the new data on the prompt fission $\gamma$ rays at thermal energy and above for those two isotopes has been categorized as the high-priority by the Nuclear Energy Agency under the Organization for Economic Co-operation and Development [4]. The majority of measurements made for the prompt $\gamma$-ray emission in fission always employed a single or a few $\gamma$-ray detectors. For example, a single $\mathrm{NaI}$ detector was used by Verbinski et al. [5] more than 40 years ago and the cerium-doped $\mathrm{LaBr}_{3}$, $\mathrm{CeBr}_{3}$, and $\mathrm{LaBr}_{3}$ detectors were used recently by Billnert et al. [1] and Oberstedt et al. [6,7].

\footnotetext{
a Corresponding author: wu24@llnl.gov
} 
Below we describe the analysis and results on the total $\gamma$-ray emission in fission measured by the DANCE array [8,9]. DANCE consists of 160 equal-volume, equal-solid-angle $\mathrm{BaF}_{2}$ detectors, covering a $4 \pi$ geometry space, and is located at the Los Alamos Neutron Science Center (LANSCE). Several unique features exhibited by DANCE are particularly attractive for those measurements, such as the nearly $\gamma$-ray energy independence for the detection efficiency, the multiplicity response, and the peak-to-total ratio, all of which are described in detail in Refs. [1012]. For example, it enables one to measure the total $\gamma$-ray energy as a function of multiplicity. The only limitation is the energy resolution, which is about $14 \%$ for the measured total $\gamma$-ray energy. A series of measurements of the prompt $\gamma$ rays in the neutron-induced fission of ${ }^{235} \mathrm{U}$ and ${ }^{239,241} \mathrm{Pu}$, and the spontaneous fission of ${ }^{252} \mathrm{Cf}$ has been carried out recently using DANCE in coincidence with the detection of fission fragments by a compact parallel-plate avalanche counters (PPAC) [13]. The results on the measured and unfolded fission prompt $\gamma$-ray energy and multiplicity distributions for those isotopes have been published $[12,14]$. An independent analysis of the same data for ${ }^{239} \mathrm{Pu}$, by assuming a general parameterized correlation between $\mathrm{E}_{\gamma}$ and $\mathrm{M}_{\gamma}$, was presented in Ref. [15]. We also reported the total prompt $\gamma$-ray energy distributions for those isotopes, obtained by unfolding the measured two-dimensional spectrum of total $\gamma$-ray energy vs multiplicity [16]. This unfolding procedure and the implication on the $\gamma$ heating in nuclear reactors are described.

\section{Experiments and data analysis}

The measurements of the prompt $\gamma$ emission in the neutron-induced fission of ${ }^{235} \mathrm{U}$ and ${ }^{239,241} \mathrm{Pu}$ as well as the spontaneous fission in ${ }^{252} \mathrm{Cf}$ were performed at the Lujan Center of LANSCE. The experimental setup and the data analysis have been described in detail in our early publications [12,14-16]. A brief summary of the experiments is given here. For the neutron-induced fission experiment, neutrons with energies from thermal up to several hundred $\mathrm{keV}$ were produced first by bombarding an $800-\mathrm{MeV}$ proton beam at a repetition rate of $20 \mathrm{~Hz}$ on a tungsten target then moderated by water. The prompt $\gamma$ rays emitted in fission were detected by the DANCE array in coincidence with the detection of fission fragments by a compact PPAC [13]. More than $10^{6}$ fission events with at least one $\gamma$ ray detected by DANCE were collected for all isotopes studied. The threshold for detecting $\gamma$-ray energy by DANCE was set to $150 \mathrm{keV}$. The summed energy of all $\gamma$ rays detected by DANCE within a time window of $40 \mathrm{~ns}$ was defined as the total prompt $\gamma$-ray energy $\left(\mathrm{E}_{\gamma, \text { tot }}\right)$ in fission for a given event. With this time window extended to $100 \mathrm{~ns}$, little change was observed for the $E_{\gamma, \text { tot }}$ spectrum [15]. The possible background contribution to $E_{\gamma, \text { tot }}$ is due to capture of thermalized prompt fission neutrons by Ba isotopes, which is on the order of $\mu$ s and too long in the time scale for prompt $\gamma$ rays. Additional suppression of neutron contribution is made by placing a gate on the pulse height spectrum of PPAC in addition to the 8-ns gate on the time spectrum between PPAC and DANCE, show in Fig. 1. All the offline data analysis was carried out using the code, FARE [17]. Note that both DANCE and PPAC have a similar intrinsic time resolution of $\sim 1.2 \mathrm{~ns}$ [13]. The total $\gamma$-ray multiplicity $\left(\mathrm{M}_{\gamma}\right)$ in fission is established not according to the number of detectors observing the $\gamma$ ray, but instead according to the number of clusters by grouping adjacent detectors catching the $\gamma$ ray in the same time window. This counting method for $\mathrm{M}_{\gamma}$ is closer to the simulated results using the $\gamma$-ray calibration sources [10-12]. In addition, the nearly $\gamma$-ray energy independence of the DANCE response to $\mathbf{M}_{\gamma}$, indicated by the numerical simulations, enables one to unfold approximately the measured $\mathbf{M}_{\gamma}$ distribution in fission for the first time $[12,14]$.

Corrections have to be made to the measured $\mathrm{E}_{\gamma, \text { tot }}$ distribution to obtain the physical one, which would be useful for the applications. This can be accomplished by unfolding the two-dimensional spectrum of $\mathrm{E}_{\gamma, \text { tot }}$ vs $\mathrm{M}_{\gamma}$. The two-dimensional unfolding is necessary because of the strong dependence of $\mathrm{E}_{\gamma, \text { tot }}$ on $\mathrm{M}_{\gamma}$. It is numerically implemented by adopting the iterative Bayesian 
method [18-20]. The DANCE response matrix for $\mathrm{E}_{\gamma, \text { tot }} \mathrm{vs}_{\gamma}$ is simulated using the GEANT4 [21] geometrical model of both DANCE and PPAC [12,14,22]. To make sure this two-dimensional response matrix has a sufficient coverage of the phase space beyond the measured one, the value of $\mathrm{M}_{\gamma}$ up to 25 and $\mathrm{E}_{\gamma, \text { tot }}$ up to $40 \mathrm{MeV}$ are included. The $\mathrm{E}_{\gamma, \text { tot }}$ has a bin size of $200 \mathrm{keV}$ and an energy threshold of $150 \mathrm{keV}$. So the response matrix has a size of $200 \times 25$.

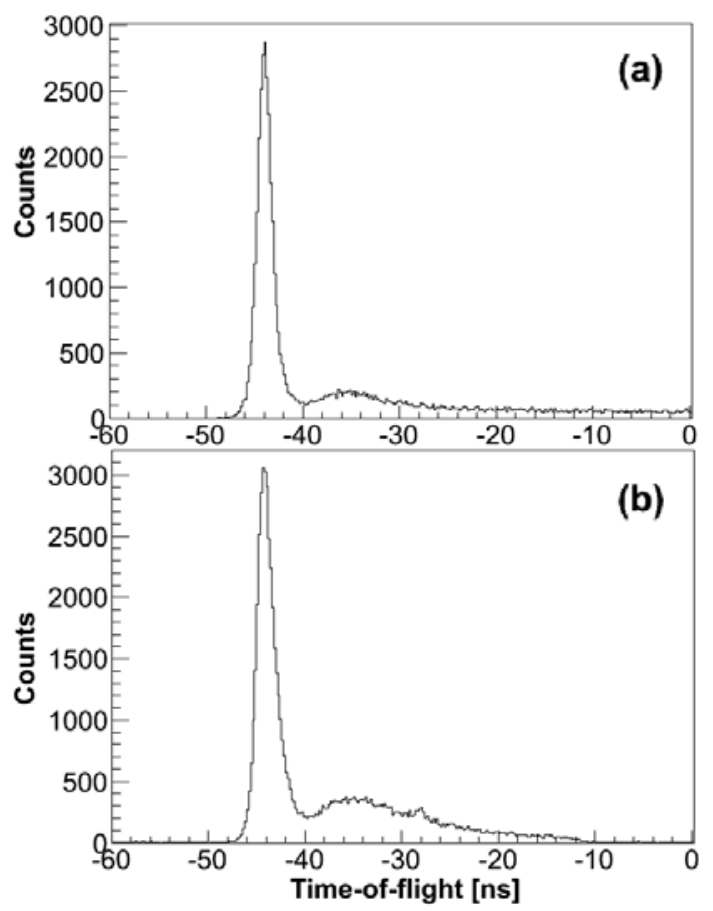

Figure 1. Time difference between $\gamma$ rays detected by DANCE and fission fragments detected by PPAC for (a) ${ }^{235} \mathrm{U}$ and (b) ${ }^{241} \mathrm{Pu}$ experiments with an achieved time resolution of $\sim 1.7 \mathrm{~ns}$. The bump next to the peak is related to events with ambiguous correlation between DANCE and PPAC.

For any given grid point $\left(\mathrm{E}_{\gamma, \text { tot }}, \mathrm{M}_{\gamma}\right)$ in the response matrix, a two-dimensional DANCE response matrix of a size of $200 \times 25$ is generated using GEANT4 with a given assembly of no more than 20,000 samples. Note that the DANCE response to the total prompt $\gamma$-ray is relatively insensitive to the content of $\gamma$ rays for a given sample since the $\gamma$-ray detection efficiency ( 84 to $88 \%$ ) and the peak-to-total ratio $(\sim 55 \%)$ remain nearly constant for the $\gamma$-ray energy ranging from $150 \mathrm{keV}$ to 10 $\mathrm{MeV}$ [10-12]. Each sample has a matching number of $\gamma$ rays to $\mathrm{M}_{\gamma}$, selected randomly according to the unfolded $\gamma$-ray energy distributions $[12,14]$ with the condition on the total $\gamma$-ray energy that is equal to $\mathrm{E}_{\gamma, \text { tot }} \pm 100 \mathrm{keV}$. This simulation is repeated for all the grid points within the lower and upper bound of $\mathrm{E}_{\gamma, \text { tot }}$ for a given $\mathrm{M}_{\gamma}$, established by this random sampling technique.

The resulting $\left(\mathrm{E}_{\gamma, \text { tot }}, \mathrm{M}_{\gamma}\right)$ DANCE response matrix consists of $~ 3300$ two-dimensional matrices with a size of $200 \times 25$ each. This numerically simulated DANCE response matrix is unique for each isotope studied, and was used to unfold the measured two-dimensional spectrum of $\mathrm{E}_{\gamma, \text { tot }} \mathrm{vs}_{\gamma}$ into a physical one using the iterative Bayesian method. During the iteration stage, a single factor was applied to and varied for the response matrix at any given grid point.

\section{Results and discussions}


Typically it takes about 30 iterations to reach the convergence in the unfolding of the twodimensional spectrum of $\mathrm{E}_{\gamma, \text { tot }} \mathrm{vs} \mathrm{M}_{\gamma}$ using the Bayesian method. The results for the neutroninduced fission in ${ }^{239} \mathrm{Pu}$ are shown in Fig. 2 where the unfolded $\mathrm{E}_{\gamma, \text { tot }} \mathrm{vs} \mathrm{M}_{\gamma}$ spectrum together with the measured one are given. In addition, the comparisons of the projected $\mathrm{E}_{\gamma, \text { tot }}$ and $\mathrm{M}_{\gamma}$ distributions between the unfolded and measured ones are also given. The general trend of the results is that the mean value and the width of projected $\mathrm{E}_{\gamma, \text { tot }}$ and $\mathrm{M}_{\gamma}$ distributions increases noticeably after the unfolding.
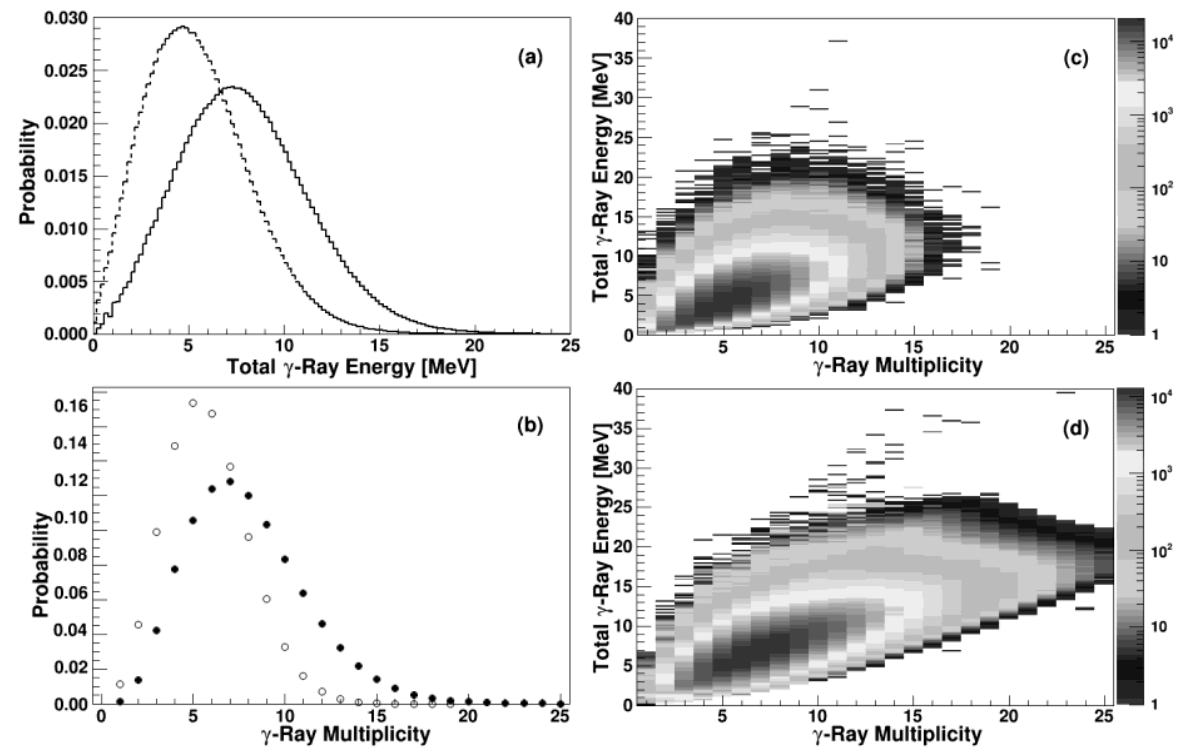

Figure 2. Shown in panel (c) and (d), respectively, are the measured and unfolded total prompt $\gamma$-ray energy vs. multiplicity distribution for the neutron-induced fission of ${ }^{239} \mathrm{Pu}$. Comparison of the projected total $\gamma$-ray energy and multiplicity distributions between measured (dashed line, open circles) and unfolded one (solid line, filled circles) are given in panels (a) and (b), respectively.

Given in Table 1 is the comparison of $\left\langle\mathrm{M}_{\gamma}\right\rangle$ derived from the unfolded $\mathrm{M}_{\gamma}$ distribution between the recent work (2-D) and the early one using the one-dimension unfolding technique [14] for all isotopes studied. For ${ }^{235} \mathrm{U}$, the recent (2-D) mean value of 7.35 is 0.37 higher than 6.98 in the earlier $1-\mathrm{D}$ work. However, the latter value is known to be underestimated by about 0.30 . Since these values were derived from the same data set, this consistency in the derived mean $\mathrm{M}_{\gamma}$ from both the one- and two-dimensional unfolding techniques gives us a certain confidence in the validity of our work. This trend is the same for ${ }^{239,241} \mathrm{Pu}$ and ${ }^{252} \mathrm{Cf}$. The comparison with other measurements and evaluations also is given in Table 1. Our measured $\left\langle\mathrm{M}_{\gamma}\right\rangle$ 's for all isotopes studied are consistently higher than the weighted-average of earlier measurements [23] by $\sim 10 \%$ except for the most recent measurements $[1,6,7]$, where their measured $\left\langle\mathrm{M}_{\gamma}\right\rangle$ is $\sim 11 \%$ greater than ours for ${ }^{235} \mathrm{U}$ but near in agreement with ours for both ${ }^{241} \mathrm{Pu}$ and ${ }^{252} \mathrm{Cf}$. Moreover, ours are consistent with the evaluated data listed in ENDF/B-VII.1 [24]. The uncertainty for our derived $\left\langle\mathrm{M}_{\gamma}\right\rangle$ has an upper bound of about 0.30.4 or $\sim 5 \%$.

The comparison of $\left\langle\mathrm{E}_{\gamma, \text { tot }}\right\rangle$ between our measurements and previous ones is given in Table 2. For ${ }^{235} \mathrm{U}$, the recent (2-D) derived mean $\mathrm{E}_{\gamma, \text { tot }}$ of $8.35 \mathrm{MeV}$ is higher than $6.53(20) \mathrm{MeV}$, the weighted average of previous measurements [23], and $6.60 \mathrm{MeV}$, the evaluated data listed in ENDF/B-VII.1. It also is higher than $6.92(9) \mathrm{MeV}$, the most recent measurement [6]. The same comparisons are also made for the neutron-induced fission in ${ }^{239,241} \mathrm{Pu}$ and the spontaneous fission in ${ }^{252} \mathrm{Cf}$. Our measured $\left\langle\mathrm{E}_{\gamma, \text { tot }}\right\rangle$ are consistently higher than the previous ones $[1,6,7,23]$ by $\sim 20 \%$ for all isotopes 
studied. The uncertainty for our derived $\left\langle\mathrm{E}_{\gamma, \text { tot }}\right\rangle$ is dominated by the systematic error and roughly estimated to be better than $5 \%$, assuming a similar uncertainty to that of the derived $\left\langle\mathrm{M}_{\gamma}\right\rangle$.

Table 1. Comparison of the mean $\mathrm{M}_{\gamma}$ between our recent measurements and previous ones for the neutroninduced fission of ${ }^{235} \mathrm{U}$ and ${ }^{239,241} \mathrm{Pu}$ as well as the spontaneous fission of ${ }^{252} \mathrm{Cf}$.

\begin{tabular}{ccccccc}
\hline Isotope & 2-D & 1-D & Ref. 15 & $\begin{array}{c}\text { ENDF/B- } \\
\text { VII.1 }\end{array}$ & Ref. 23 & Refs. 1, 6, 7 \\
\hline${ }^{{ }^{235} \mathbf{U}}$ & 7.35 & 6.95 & & 7.04 & $6.60(10)$ & $8.19(11)$ \\
${ }^{239} \mathbf{P u}$ & 7.93 & 7.50 & 7.15 & 7.78 & $7.06(20)$ & \\
${ }^{241} \mathbf{P u}$ & 7.97 & 7.50 & & 8.18 & & $8.21(9)$ \\
${ }^{252} \mathbf{C f}$ & 8.75 & 8.16 & & & $7.98(40)$ & $8.30(8)$ \\
\hline
\end{tabular}

An independent analysis of the same DANCE data for ${ }^{239} \mathrm{Pu}$ by assuming a very general parameterized correlation between $\mathrm{E}_{\gamma}$ and $\mathrm{M}_{\gamma}$ has been carried out by Ullmann et al. [15], which yields the $\left\langle\mathrm{E}_{\gamma, \text { tot }}\right\rangle=7.46 \mathrm{MeV}$ and $\left\langle\mathrm{M}_{\gamma}\right\rangle=7.15$. The $\left\langle\mathrm{E}_{\gamma, \text { tot }}\right\rangle$, derived from the $\mathrm{E}_{\gamma, \text { tot }}$ distribution, agrees within $6 \%$ of that obtained by using the 2-D unfolding technique. This agreement is significant and indicates the importance of the correlation between $\mathrm{E}_{\gamma}$ and $\mathrm{M}_{\gamma}$ to be considered in the determination of $\left\langle\mathrm{E}_{\gamma, \text { tot }}\right\rangle$. It raises serious concern on the validity of the equation, $\left\langle\mathrm{E}_{\gamma, \text { tot }}\right\rangle=\left\langle\mathrm{E}_{\gamma}\right\rangle \times$ $\left\langle\mathrm{M}_{\gamma}\right\rangle$, which ignores the correlation between $\mathrm{E}_{\gamma}$ and $\mathrm{M}_{\gamma}$ exhibited in Fig. 2.

Table 2. Comparison of the mean $\mathrm{E}_{\gamma, \text { tot }}(\mathrm{MeV})$ between our recent measurements and previous ones for the neutron-induced fission of ${ }^{235} \mathrm{U}$ and ${ }^{236,241} \mathrm{Pu}$ as well as the spontaneous fission of ${ }^{252} \mathrm{Cf}$.

\begin{tabular}{cccccc}
\hline Isotope & 2-D & Ref. 15 & $\begin{array}{c}\text { ENDF/B- } \\
\text { VII.1 }\end{array}$ & Ref. 23 & Refs. 1, 6, 7 \\
\hline${ }^{235} \mathbf{U}$ & 8.35 & & 6.60 & $6.53(20)$ & $6.92(9)$ \\
${ }^{239} \mathbf{P u}$ & 7.94 & 7.46 & 6.74 & $6.78(10)$ & \\
${ }^{241} \mathbf{P u}$ & 8.01 & & 7.26 & & $6.41(6)$ \\
${ }^{252} \mathbf{C f}$ & 8.52 & & & $6.95(30)$ & $6.64(8)$ \\
\hline
\end{tabular}

\section{Summary}

A systematic study of the total prompt $\gamma$-ray emission in the neutron-induced fission of ${ }^{235} \mathrm{U}$ and ${ }^{239,241} \mathrm{Pu}$ as well as the spontaneous fission of ${ }^{252} \mathrm{Cf}$ has been carried out using the DANCE array together with a compact PPAC to select the fission event by detecting its fission fragments. The total $\gamma$-ray energy vs multiplicity spectrum for all fissile nuclei studied was constructed and unfolded using a two-dimensional unfolding technique, numerically implemented by adopting the iterative Bayesian method. The $\left\langle\mathrm{E}_{\gamma, \text { tot }}\right\rangle$ derived from the projected $\mathrm{E}_{\gamma, \text { tot }}$ distribution of the unfolded $\mathrm{E}_{\gamma, \text { tot }} \mathrm{vs} \mathrm{M}_{\gamma}$ spectrum is about $20 \%$ higher than the previous measurements for all fissile nuclei studied. However, it agrees reasonably well with the result derived from the analysis by considering the correlation between $\mathrm{E}_{\gamma}$ and $\mathrm{M}_{\gamma}$ in a very general parameterization manner. In addition, the measured total prompt $\gamma$-ray energy vs multiplicity spectrum in fission enables one to evaluate the variance in addition to the average value of the energy deposited in a reactor core by the prompt fission $\gamma$ rays. This may improve our understanding of the $\gamma$ heating in many applications involving nuclear fission.

\section{Acknowledgement}

This work benefited from the use of the LANSCE accelerator facility as was performed under the auspices of the US Department of Energy by Lawrence Livermore National Security, LLC under 
contract DE-AC52-07NA27344 and by Los Alamos National Security, LLC under contract DEAC52-06NA25396. Partial funding is gratefully acknowledged from the U.S. DOE/NNSA Office of Defense Nuclear Nonproliferation Research and Development. All isotopes used in the measurements were obtained from Oak Ridge National Laboratory.

\section{References}

1. R. Billnert, F.-J. Hambsch, A. Oberstedt, and S. Oberstedt, Phys. Rev. C 87, 024601 (2013)

2. G. Rimpault, in Nuclear Data Needs for Generation IV, edited by P. Rullhusen (World Scientific, Antwerp, 2006), p. 46

3. G. Rimpault, A. Courcelle, and D. Blanchet, comment to HPRL: ID H.3 and H.4

4. Nuclear Data High Priority Request List of the NEA (Req. ID: H.3, H4) http://www.oecdnea.org/dbdata/hprl/hprlview.pl?ID $=421$ and http://www.oecdnea.org/dbdata/hprl/hprlview.pl?ID $=422$

5. V.V. Verbinski, H. Weber, and R.E. Sund, Phys. Rev. C 7, 1173 (1973)

6. A. Oberstedt et al., Phys. Rev. C 87, 051602(R) (2013)

7. A. Oberstedt et al., Phys. Rev. C 90, 024618 (2014)

8. M. Heil, R. Reifarth, M. M. Fowler, R. C. Haight, F. Kappeler, R. S. Rundberg, E. H. Seabury, J. L. Ullmann, and K. Wisshak, Nucl. Instrum. Methods Phys. Res. A 459, 229 (2001)

9. R. Reifarth et al., IEEE Transactions on Nuclear Science 53, 880 (2006)

10. R. Reifarth et al., Los Alamos National Laboratory, LA-UR-01-4185 (2001)

11. R. Reifarth et al., Los Alamos National Laboratory, LA-UR-03-5560 (2003)

12. A. Chyzh, C. Y. Wu et al., Phys. Rev. C 85, 021601(R) (2012)

13. C.Y. Wu, A. Chyzh, E. Kwan, R. Henderson, J. Gostic, D. Carter, T. Bredeweg, A. Couture, M. Jandel, J. Ullmann, Nucl. Instrum. Methods Phys. Res. A 694, 78 (2012)

14. A. Chyzh, C.Y. Wu et al., Phys. Rev C 87, 034620 (2013)

15. J.L. Ullmann et al. Phys. Rev. C 87, 044607 (2013)

16. A. Chyzh, C. Y. Wu et al., Phys. Rev. C 90, 014602 (2014)

17. M. Jandel, T.A. Bredeweg, A. Couture, J.M. O’Donnel, and J.L. Ullmann, Los Alamos National Laboratory, LA-UR-12-21171 (2012)

18. G. D’Agostini, Nucl. Instrum. Methods Phys. Res. A 362, 487 (1995)

19. G. D’Agostini, arXiv:1010.0612 (2010)

20. T.J. Adye, arXiv:1105.1160 (2011)

21. S. Agostinelli et al., Nucl. Instrum. Methods Phys. Res. A 506, 550 (2003)

22. M. Jandel et al., Nucl. Instrum. Methods Phys. Res. B 261, 1117 (2007)

23. T.E. Valentine, Ann. Nucl. Energy 28, 191 (2001) and references therein

24. M.B. Chadwick et al., Nucl. Data Sheets 112, 2887 (2011) 